Commentary on Susan Hurley's 'The Shared Circuits Model'. To appear in the Behavioral and Brain Sciences

Abstract - 73
Main text - 1080
References - 106

Total - 1259

\title{
IMITATION AS A CONJUNCTION
}

\author{
Cecilia Heyes \\ Department of Psychology, University College London, Gower Street, London \\ WC1 6BT, United Kingdom \\ $44(0) 2076795378$ \\ c.heyes@ucl.ac.uk
}

http://www.psychol.ucl.ac.uk/celia.heyes/netintro.htm

\begin{abstract}
The conjunctive conception takes imitation to be a combination of observational learning and copying. In the target article, and elsewhere, this conception generates problems in 1) explaining the copying of intransitive actions, 2) elucidating the potential functions of imitation, and 3) recognising when the correspondence problem has been avoided rather
\end{abstract}


than solved. Hurley's careful use of subpersonal and personal levels of explanation shows us how to tackle these and other questions about imitation. 
Rarely does an empirically-minded philosopher - or, for that matter, a scientific specialist - achieve the kind of breadth, balance and penetration of a scientific literature evident in Hurley's review of research on imitation. In nearly all respects, the SCM's embeddedness in this literature is a huge asset, to the model and to the field. However, she adopted one, central feature of this literature - a complex, conjunctive conception of imitation - that is problematic.

Hurley takes imitation to be a phenomenon in which action observation causes the observer both 1) to learn an instrumental relationship between a body movement and its effect, and thereby 2) to perform the observed body movement. Here I shall call the first of these conditions 'observational learning' and the second 'copying'. An alternative conception of imitation, increasingly common in the literature, distinguishes more firmly between observational learning and copying, and calls the latter 'imitation'.

Empirical evidence and everyday experience confirm that observational learning and copying can occur independently. As a spectator at Wimbledon, I can learn about the relationship between body movement dynamics and ball dynamics without being able to copy an ace serve. Conversely, when we are talking, I may copy the way in which you tug your earlobe or jiggle your foot, without knowing what, if any, are the ‘effects’ of these actions (Chartrand \& Bargh 1999).

There is no doubt that observational learning and copying can be linked in the manner assumed by the conjunctive conception of imitation; observing your action sometimes 
both enables me to copy, by showing me a new sequence of movements, and motivates me to realise this potential, by showing me that the sequence has a particular, desirable outcome. And the functional properties of this conjunction have long been of interest to researchers seeking the origins of distinctively human sociocognitive abilities. Hurley’s decision to root the SCM model in the conjunctive conception of imitation was, therefore, consistent both with the purposes of the SCM, and with the approach of many scientists in the field. But it led to some problems that might be avoided in future development of the model if observational learning were more firmly distinguished from copying.

The first problem, concerning the explanatory range of the SCM, is openly acknowledged in the target article. Because the SCM assumes that learning about means-ends relationships is an essential component of imitation, and because it treats 'ends' as effects of body movement on the world (note the "external feedback loop" in Layer 1), it is not clear "Whether SCM can extend from instrumental to expressive action" (p.36). In other words, the model does not currently account for copying of intransitive actions, such as facial and hand gestures; a type of copying that includes many paradigmatic examples of imitation in the colloquial sense, and appears to be more closely related than copying of instrumental actions to the functioning of human mirror system (e.g. Fadiga, Fogassi, Pavesi \& Rizzolatti 1995).

The second problem relates to the functions of imitation. Section 1.3 of the target article begins with a finely-turned pair of questions: “Does the development of either language or mindreading depend on imitation? If so, at what levels of description and in what 
senses of 'depend'?”. (Many of us, tempted to make crude claims about the evolutionary-developmental consequences of imitation, would do well to use this quote as a banner screensaver.) However, having stated the question so clearly, Hurley is compelled by the conjunctive conception of imitation to be, on occasions, less lucid in suggesting and endorsing relationships between imitation and other sociocognitive functions. Take language as an example. She distinguishes four senses in which language may depend on imitation: two, relating to means and ends, that connect language with observational learning (and possibly with all goal-directed action) but not with copying, and two, relating to common coding and parsing of body movement sequences, that connect language with copying, but not with observational learning. When copying and observational learning are firmly distinguished, it is possible to see that one or the other may be involved in the evolution and/or development of language, but the conjunctive conception of imitation gives the impression that, if one is involved, then so is the other.

The third problem is that the conjunctive conception of imitation tends to make researchers lose sight of the correspondence problem; to forget that copying often requires the observer to map visual information from action observation onto matching motor output, under conditions where it is far from obvious how she could have acquired the information necessary to achieve this mapping. The classic examples are facial movements; the sensory input that I receive when I see you, for example, curling your lip, is in a different modality and coordinate frame from the sensory feedback I receive when I produce the same gesture (Brass \& Heyes 2005). 
The SCM does not neglect the correspondence problem. Indeed, it is the principal function of Layer 3 to solve this problem, and Hurley and I agree that the solution comes from associative learning (e.g. Heyes 2001; Catmur, Walsh \& Heyes 2007). However, under the influence of the conjunctive view of imitation, which focuses attention on the impetus or motivation for copying, rather than on its epistemic base, Hurley lets other models off the hook a bit too easily. Explaining imitation with reference to mirror neurons, or to the common codes postulated by ideomotor theory, does indeed 'avoid' the correspondence problem, and that is entirely reasonable given that neither mirror neuron research nor ideomotor theory is intended primarily to explain copying/imitation. However, it is important to recognise that avoiding is very different from solving. Mirror neurons and common codes merely move the correspondence problem from the personal to the subpersonal level. Instead of asking how the observer knows that lip-curling is the same when observed and executed, we have to ask how mirror neurons or common codes get this information.

One of the greatest strengths of Hurley's article is the way that it gently but firmly encourages those of us involved in research on imitation to clean up our act with respect to levels of explanation. The SCM is a model, not just of imitation-related functions, but of how to respect the three boundaries between subpersonal (neurological and function) and personal levels of explanation. By teasing them apart, Hurley reveals the complex and sometimes tenuous nature of many pre-existing hypotheses, illuminates questions for 
further empirical research, and leaves us no excuse for muddled thinking about the mechanisms and functions of imitation.

\section{References}

Brass, M. \& Heyes, C. M. (2005). Imitation: Is cognitive neuroscience solving the correspondence problem? Trends in Cognitive Sciences, 9, 489-495.

Catmur, C., Walsh, V. \& Heyes, C. M. (2007) Sensorimotor learning configures the human mirror system. Current Biology, 17, 1527-1531.

Chartrand, T. L., \& Bargh, J. A. (1999). The chameleon effect: The perception-behaviour link and social interaction. Journal of Personality \& Social Psychology, 76, 893-910.

Fadiga, L., Fogassi, L., Pavesi, G., \& Rizzolatti, G. (1995). Motor facilitation during action observation: A magnetic stimulation study. Journal of Neurophysiology, 73, 26082611.

Heyes, C. (2001). Causes and consequences of imitation. Trends in Cognitive Sciences. 5, 253-261. 